\title{
Erratum to: Effects of Obovatol on GSH Depleted Glia-Mediated Neurotoxicity and Oxidative Damage
}

\author{
Moonhee Lee • Byoung-Mog Kwon • Kyoungho Suk • \\ Edith McGeer $\cdot$ Patrick L. McGeer
}

Published online: 11 August 2011

(C) Springer Science+Business Media, LLC 2011

\section{Erratum to: J Neuroimmune Pharmacol}

\section{DOI 10.1007/s11481-011-9300-9}

Due to a publisher error, the name of Dr. Byoung-Mog Kwon was misspelled in the original publication. Dr. Kwon's name is reproduced correctly above.

The online version of the original article can be found at http://dx.doi. org/10.1007/s11481-011-9300-9.

M. Lee $\cdot$ E. McGeer P. L. McGeer $(\bowtie)$

Kinsmen Laboratory of Neurological Research, University of British Columbia,

2255 Wesbrook Mall,

Vancouver, British Columbia, Canada V6T 1 Z3

e-mail: mcgeerpl@interchange.ubc.ca

\section{B.-M. Kwon}

Laboratory of Chemical Biology and Genomics,

Korea Research Institute of Bioscience and Biotechnology,

University of Science and Technology,

Daejeon, Korea

\section{K. Suk}

Department of Pharmacology, Kyungpook National University, School of Medicine, Brain Science and Engineering Institute,

Daegu, Korea 